\title{
Comparing the reflectivity of ungrouped carbonaceous chondrites with those of short-period comets like 2P/Encke
}

\author{
Safoura Tanbakouei ${ }^{1,2}$, Josep M. Trigo-Rodríguez ${ }^{1,2}$, Jürgen Blum ${ }^{3}$, Iwan Williams ${ }^{4}$, and Jordi Llorca ${ }^{5}$ \\ ${ }^{1}$ Institute of Space Sciences (ICE-CSIC), Campus UAB, C/ Can Magrans s/n, 08193 bellaterra (Barcelona), Catalonia, Spain \\ e-mail: tanbakouei@ice.csic.es \\ 2 Institut d'Estudis Espacials de Catalunya (IEEC), C/ Gran Capità, 2-4, Ed. Nexus, desp. 201, 08034 Barcelona, Catalonia, Spain \\ e-mail: trigo@ice.csic.es \\ ${ }^{3}$ Institut für Geophysik und extraterrestrische Physik, Technische Universität Braunschweig, Mendelssohnstr. 3, 38106 Braunschweig, \\ Germany \\ e-mail: j.blum@tu-bs.de \\ ${ }^{4}$ School of Physics and Astronomy, Queen Mary, University of London, Mile End Rd. London E1 4NS, UK \\ ${ }^{5}$ Institute of Energy Technologies, Department of Chemical Engineering and Barcelona Research Center in Multiscale Science and \\ Engineering, Universitat Politècnica de Catalunya- BarcelonaTech, Catalonia, Spain
}

Received 20 March 2020 / Accepted 6 July 2020

\begin{abstract}
Aims. The existence of asteroid complexes produced by the disruption of these comets suggests that evolved comets could also produce high-strength materials able to survive as meteorites. We chose as an example comet 2P/Encke, one of the largest object of the so-called Taurid complex. We compare the reflectance spectrum of this comet with the laboratory spectra of some Antarctic ungrouped carbonaceous chondrites to investigate whether some of these meteorites could be associated with evolved comets.

Methods. We compared the spectral behaviour of 2P/Encke with laboratory spectra of carbonaceous chondrites. Different specimens of the common carbonaceous chondrite groups do not match the overall features and slope of the comet 2P/Encke. By testing anomalous carbonaceous chondrites, we found two meteorites: Meteorite Hills 01017 and Grosvenor Mountains 95551, which could be good proxies for the dark materials that formed this short-period comet. We hypothesise that these two meteorites could be rare surviving samples, either from the Taurid complex or another compositionally similar body. In any case, it is difficult to get rid of the effects of terrestrial weathering in these Antarctic finds, and further studies are needed. A future sample return from the so-called dormant comets could also be useful to establish a ground truth on the materials forming evolved short-period comets.

Results. As a natural outcome, we think that identifying good proxies of $2 \mathrm{P} /$ Encke-forming materials might have interesting implications for future sample-return missions to evolved, potentially dormant, or extinct comets. Understanding the compositional nature of evolved comets is particularly relevant in the context of the future mitigation of impact hazard from these dark and dangerous projectiles.
\end{abstract}

Key words. comets: general - meteorites, meteors, meteoroids - techniques: spectroscopic - ultraviolet: planetary systems

\section{Introduction}

The comet 2P/Encke (hereafter Encke) is a $4.8 \mathrm{~km}$-sized active comet that was discovered in 1786. It has an unusual $3.3 \mathrm{yr}$ orbit, and is one of the largest known objects in the Taurid complex (hereafter TC) (Clube \& Napier 1984; Asher et al. 1993). Dynamically the TC was thought to have its origins within the main asteroid belt (Hsieh \& Jewitt 2006; Jewitt 2012), but the discovery of tens of asteroids in similar orbits to the meteoroid complex suggest its origins lie in the disruption of a more eccentric and large comet. A formation scenario in which Encke is one of many fragments resulting from the break-up of a much larger comet was proposed by Clube \& Napier (1984) and later discussed by Asher et al. (1993) and Babadzhanov et al. (2008). The importance of the study of the TC under an impact hazard perspective seems obvious, particularly with the recent discovery of challenging dormant comets in near-Earth space (Binzel et al. 2015) that might be well exemplified by the Earth's encounter with the challenging 2015 TB145 (Müller et al. 2017; Micheli et al. 2018). On the other hand, a potentially hazardous asteroid (PHA), 2008 XM1, was recently identified as one of the objects producing meteoroids dynamically associated with this complex (Madiedo et al. 2014). Moreover, Earth's encounter with the TC debris has been proposed as the source of a catastrophic aerial blast that occurred around $12900 \mathrm{BC}$, which promoted a return to ice age conditions for $\sim 1300 \mathrm{yr}$ (Napier 2010). On the other hand, recent meteor studies have suggested that bright Taurid fireballs exhibit finite ending masses, so that there is some chance that meteorite collections contain free-delivered samples of 2P/Encke or some other members of the TC (Madiedo et al. 2014; Spurnỳ et al. 2017).

Due to gravitational interactions with the terrestrial planets, Encke's orbit is far from Jupiter's control, and this results in a very smooth orbital evolution (see e.g. Levison et al. 2006). The properties of Encke are quite distinct compared to other objects in the Solar System (Boehnhardt et al. 2008). Compared to the available spectra of other cometary nuclei, Encke's spectrum is rather typical, despite its very peculiar orbit. It is also important to remark that Encke exemplifies a most challenging hazardous body, because it has a very low albedo, reflecting only $4.6 \%$ of the light it receives in the visible range (Tubiana et al. 2015).

It might be surprising that most members of the TC are asteroidal in nature, but it is probably a natural outcome of the evolution of short-period comets (Binzel et al. 2015; 
Jewitt 2008). The volatile content for each one of these Taurid members was perhaps inhomogeneous, or possibly affected by other processes that extinguished their volatile content, for example close approaches to the Sun or impact gardening (Beitz et al. 2016). Obviously, these new objects found suggest that the comet Encke could be part of a transitional and inhomogeneous progenitor, coming from a formation region in the asteroid/comet boundary (Hsieh \& Jewitt 2006; Hsieh 2017). In such a sense, a recent study of the reflectance spectra of the largest asteroids dynamically associated with Encke revealed that their spectral characteristics are linked to the $\mathrm{S}$ taxonomic complex (Popescu et al. 2014). The Comet Encke has a visible spectrum, similar to other cometary nuclei and closer to D-type asteroids (Raponi et al. 2020), as it was also suggested from the behaviour of ungrouped Tagish Lake carbonaceous chondrites (Hiroi et al. 2001). Such a result points towards a heterogeneous comet progenitor, which after disruption produced the known PHAs on Apollo-type orbits, some of them exhibiting ordinary chondritelike mineralogy. It could have deep implications for impact hazard, but given the degree of collisional processing, we cannot discard the concept that short-period comets suffered the implantation of foreign rocky projectiles that contributed to the modification of their surfaces and produced high-strength meteoroids when disrupted (Kresak 1978). We should also mention in this context that the Tunguska airblast on June 30, 1908 was initially associated with the TC (Trigo-Rodríguez \& Williams 2017). Sekanina (1998) later discissed such a cometary nature with the argument that the fireball had a disruption behaviour characteristic of a stony asteroid. If the bodies forming the TC are so heterogeneous in nature, it could make room for both scenarios being correct, after all. In any case, it has been accepted that a hydrated asteroid should progressively deplete its volatiles that are mostly bound in minerals rather than forming ices (Trigo-Rodríguez 2015; Trigo-Rodríguez et al. 2019). In fact, most TC members behave like asteroids and do not exhibit any cometary activity at all. In this sense, we are interested in testing whether some carbonaceous chondrites (CC) could be good proxies of the reflectance properties of the comet Encke. Current formation models predict that short-period comets can only stay active for a relatively short time (or number of perihelion passages), because their high internal lithostatic compression hardens the material so much that activity stops when a thin surface layer has been emitted (Gundlach \& Blum 2016).

The reflectance spectrum of Encke typically exhibits a moderate red slope and is otherwise featureless (Luu \& Jewitt 1990). To our knowledge, it has so far not been possible to find a meteorite proxy with a similar reflectance behaviour. Consequently, the main goal of our work is to identify samples in our meteorite collections with reflectance characteristics similar to those of comet Encke. We consider Encke as a case study to promote studies with the aim of establishing a link between short-period comets using remote sensing and laboratory studies of meteorites.

\section{Technical procedure and sample selection}

We obtained reflectance spectra of the meteorites described in Tables 1 and 2, using the procedure described in previous work (Trigo-Rodriguez et al. 2014). Polished sections of the selected meteorites were measured at UPC, using a Shimadzu UV3600 ultraviolet to near-infrared (UV-Vis-NIR) spectrometer. The spectrometer diffraction-limited illumination originates from one of two lamps and passes through a variable slit, it is then filtered with a grating to select the desired wavelength, and
Table 1. List of CR, CM and CK carbonaceous chondrites that were compared to the spectrum of comet $2 \mathrm{P} /$ Encke in this work.

\begin{tabular}{ccc}
\hline \hline Meteorites & Group & Mass $(\mathrm{g})$ \\
\hline QUE 90355 & CM2 & 32.4 \\
Murchison & CM2 & $\sim 100000$ \\
EET 92159 & CR2 & 67.6 \\
LAP 02342 & CR2 & 42.4 \\
Renazzo & CR2 & $\sim 1000$ \\
PCA 82500 & CK4-5 & 90.9 \\
LAR 12265 thin & CK5 & 14.3 \\
ALH 82500 & CK5 & 438 \\
\hline
\end{tabular}

Notes. The masses were taken from the Meteoritical Bulletin and denote the total mass of the meteorite.

Table 2. Ungrouped carbonaceous chondrites found in this work to possess a reasonable similarity to the spectrum of comet 2P/Encke.

\begin{tabular}{ccc}
\hline \hline Meteorites & Group & Mass (g) \\
\hline MET 01017 & CV3-an & 238.0 \\
GRO 95551 & C-ung & 213.3 \\
\hline
\end{tabular}

Notes. The masses were taken from the Meteoritical Bulletin and denote the total mass of the meteorite.

afterwards it is split into two alternating but identical beams with a chopper. Next, the beam interacts with the sample and is routed to the detector. The reference beam interacts with the material of the sample surface and then goes to the same detector (Moyano-Cambero et al. 2016). Next, the beam interacts with the sample at an angle of $8^{\circ}$ and it is later routed to the detector. The standard stage for the spectrometer is an integrating sphere with a working range in the current study of 400-900 $\mathrm{nm}$, and operated under laboratory conditions. The reflectance spectrum of Encke was taken from Tubiana et al. (2015). The spectrum was normalised to unity at $550 \mathrm{~nm}$. It is important to remark that the Encke reflectance data was removed between 570 and $620 \mathrm{~nm}$ and between 720 and $780 \mathrm{~nm}$, due to several issues related to the data reduction and some artefacts in the spectra, as explained in Tubiana et al. (2015). The meteorite specimens studied in this work and compared with Encke are listed in Tables 1 and 2 .

\section{Results and discussion}

In order to gain insight into the nature of evolved comets, we compared the reflectance spectra of CCs with the reflectance spectrum obtained for comet Encke (Tubiana et al. 2015). In our study, we included a significant number of NASA Antarctic CCs. Given the low terminal mass expected for meteorite-dropping bolides of the Taurid stream, we think that the Antarctic collection can provide the opportunity to identify the first meteorites associated with comets.

Gradie \& Veverka (1986) studied the visible to NIR spectral dependence of ordinary and carbonaceous chondrites. Their models of the surface roughness indicate that the differences found between the visual phase coefficients of S- and C-class asteroids are reflecting primarily differences in composition and not necessarily differences in surface roughness. The beam incidence angle of $8^{\circ}$ is a particular geometry that cannot be changed in our spectrometer, so we can consider that our data were 
obtained in almost specular geometry (see e.g. Britt \& Pieters 1988). These authors demonstrate that the amount of metal plays a significant role in the slope of the spectrum as we also previously discussed (Moyano-Cambero et al. 2016). On the other hand, the incidence angle might also affect the slope of the spectrum, and the overall spectral reflectivity but it has not influence on the location of absorption bands (Gradie et al. 1980).

Our reflectance spectra show features that might be different to those obtained using remote sensing. The reason for that is that each feature depth depends on many factors, for example differences in observation geometry, or the grain size, shape and roughness. In addition, we recognise that the spectral slope of carbonaceous chondrites can change from red to blue depending on whether the sample is prepared as powder or as slab. The slope also changes with grains size, packing density or observation geometry as has been studied for Murchison CC (Cloutis et al. 2018). For these reasons, the sample reflectance slope is a multivariate problem, and it provides only a first approach to look for possible proxies of solar system primitive bodies.

Comparison of the reflectance spectrum of comet Encke with those obtained for specimens of CK, CM and CR chondrites has previously been done (see e.g. Trigo-Rodriguez et al. 2014; Tanbakouei et al. 2019) (Table 1). We chose groups that exhibit significant evidence for parent-body aqueous alteration, or oxidation (Trigo-Rodríguez 2015; Trigo-Rodríguez \& Williams 2017). No clear similarity with Encke's spectrum was found as a result of such comparisons for any specimen belonging to these well-established CC groups. Then, we performed a similar oneby-one comparison with the spectra of some ungrouped CCs and found two quite reasonable matches, which we describe in the next section (Cloutis et al. 2011) (Table 2).

We did our best to get rid of terrestrial weathering, choosing those areas of the two meteorites that were minimally affected by the rusting effect. However, both ungrouped carbonaceous chondrites belong to the NASA Antarctic collection and probably spent a significant amount of time in Antarctica. Consequently, we should consider the possible impact of terrestrial weathering in both spectra. There is a possibility that the origin of the spectral slope measured for the two anomalous carbonaceous chondrites is caused by some oxides formed by terrestrial weathering. We do not think this to be the case in the areas where we collected the spectra, but if these ungrouped carbonaceous chondrites were affected by terrestrial weathering, the spectral slope could change from red to blue depending on whether the sample is prepared as powder or as slab (Cloutis et al. 2018). These authors also demonstrated that the slope is very sensitive and could change with the grain size, packing density or the observation geometry. In particular, the particle size can have a strong effect on the albedo so that a body covered in finegrained regolith with a particular phase angle has to be taken into account to make a proper comparison with the spectral properties of meteorites (Muinonen et al. 2010; Belskaya et al. 2010).

\subsection{Comparison with ungrouped carbonaceous chondrites}

The reflectance spectrum of Encke covers the full visible range from 400 to $900 \mathrm{~nm}$ on the detector. Concerning the comparison with other $\mathrm{CC}$ groups, the specimens belonging to most carbonaceous chondrite groups exhibited very different reflectance spectra. After many attempts among the meteorites studied, we found a likely reflectance similarity in one meteorite, named Meteorite Hills 01017 (MET 01017). This meteorite was initially characterised as a CV3 (possibly reduced) chondrite (Busemann et al. 2007), but due to its anomalous properties was reclassified

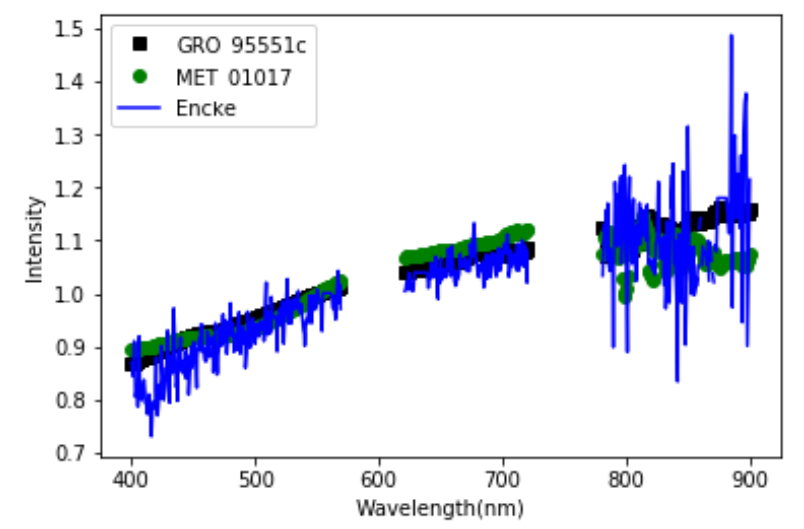

Fig. 1. Reflectance spectra from 400 to $900 \mathrm{~nm}$ of the two ungrouped carbonaceous chondrites compared with the spectrum of the comet 2P/Encke (blue line) (from Tubiana et al. 2015). MET 01017 belongs to the CV3-an achondrite group, and GRO 95551 is an ungrouped carbonaceous chondrite. All spectra were scaled and normalised to 1 at $550 \mathrm{~nm}$.

as a CV3-an (MetBull: 88, 2001). In fact, the section used in our work shows evidence of aqueous alteration with half of its exterior having a weathered fusion crust with oxidation haloes. The section also exhibits mm-sized chondrules that are quite well preserved and possesses a fractured and weathered interior. It also contains metal-rich chondrules and CAIs in a dark matrix of FeO-rich phyllosilicates (Brearley 1997; Krot et al. 2006; TrigoRodríguez 2015). Usually CV chondrites contain a $45 \%$ volume of large chondrules with an average size of $1 \mathrm{~mm}, \sim 40 \%$ of the volume of a dark matrix, and a large volume abundance of CAIs of $\sim 10 \%$ (Brearley \& Jones 1998; Scott \& Krot 2003). The matrices of CV3 chondrites are dominated by fine-grained olivine plus phyllosilicates and opaque materials such as metallic iron, sulphides and magnetite (Burbine et al. 2001). Metal grains typically range in size from 50 to $500 \mathrm{~nm}$. Sulphides, up to $\sim 200 \mu \mathrm{m}$ in size, are usually associated with metal and act as opaque material as well.

Another sample with a spectral behaviour similar to that of Encke is Grosvenor Mountains 95551 (GRO 95551). This meteorite is an ungrouped $\mathrm{CC}$, an unusual metal-rich breccia formed by two main types of clasts: chondritic and achondritic. The chondritic clasts consist of a variety of chondrules and chondrule fragments. The mean olivine composition of GRO 95551 is $\mathrm{Fa}_{1.3}$ with a range of $\mathrm{Fa}_{0.7-3.5}$ and pyroxene ranges $\mathrm{Fs}_{0.7-39.6}, \mathrm{Wo}_{0.7-1.6}$ (Weisberg et al. 2015). The meteorite is anomalous with a high metal content and some petrographic peculiarities, for example, the presence of large silicate nodules, resembling Bencubbin (Kallemeyn et al. 1978; Weisberg et al. 2001). The specimen is of interest, because it might be representative of the materials stemming from processed objects (Trigo-Rodríguez 2015; Brearley \& Jones 1998; Moyano-Cambero \& Trigo-Rodríguez 2015).

As we can see in Fig. 1, at first sight the spectral slope of the two studied meteorites is compatible with that of Encke. The spectrum of the comet shows a shallower slope above $800 \mathrm{~nm}$. However, as we explain in Sect. 2, our reflectance spectra were taken under a beam angle of $8^{\circ}$. Thus we need to recognise that it implies a significant limitation in the comparison with remote sensing data, particularly in reference to the slope. That beign said, the spectra of the comet is similar to the spectra of primitive asteroids and it seems strange that there is no evidence, even when it could be scarce in meteorite collections. It is similar to the spectra of primitive asteroids, which are believed to be associated with CCs (Tubiana et al. 2015). Figure 1 shows that 
Table 3. The location of the main absorption bands in the two ungrouped $\mathrm{CC}$ meteorites and their mineral assignments.

\begin{tabular}{ccc}
\hline \hline Meteorites & Absorption band (nm) & Mineral \\
\hline MET 01017 & 495 & Olivine \\
& 665 & Magnetite/maghemite \\
& $700-720$ & Serpentine (phyllosilicates) \\
& 850 & Olivine \\
& 875 & Orthopyroxene \\
\hline GRO 95551 & 470 & Olivine \\
& 665 & Mgnetite/maghemite \\
& 710 & Serpentine (phyllosilicates) \\
& 850 & Olivine \\
\hline
\end{tabular}

References. Cloutis et al. (2012b,c); Trigo-Rodriguez et al. (2014); Weisberg et al. (2015).

both ungrouped CC samples and comet Encke exhibit a common absorption feature at $500 \mathrm{~nm}$ (Table 3). From the spectral similarities, a tentative link between Encke, MET 01017, and GRO 95551 can be made. It is interesting that ungrouped carbonaceous chondrites have highly variable spectral properties and that the surface of the comet Encke was subjected to significant changes due to solar heating (Tubiana et al. 2015).

Weisberg et al. (2015) showed the similarities of Bencubbin and GRO 95551, but they are different in olivine compositions, oxygen isotope compositions, and the presence of interstitial sulfides, and also in the siderophile element compositions of the metal (Weisberg et al. 2015). The siderophile element composition of GRO 95551 is closer to that of ALH 85085 than to that of Bencubbin (Kallemeyn 2000). There are many of the smaller non-spherical objects in GRO 95551 that show the texture of chondrules. The lack of a matrix in GRO 95551 could be a sign of rapid accretion, with significant thermal processing (Metzler 2012). Obviously some of these features could be also the consequence of shock processing and/or heating in close approaches to the Sun (Delbo et al. 2014).

Concerning the reflectance spectra of MET 01017, a weaker absorption feature around $470-520 \mathrm{~nm}$ can also be seen, and it and appears to be compatible with a weak feature also found in the Encke reflectance spectrum (Fig. 2). It is important to consider that comet surfaces are probably extensively processed due to solar heating and collisional gardening (Trigo-Rodríguez 2015). In addition to the reflectance data, additional evidence was recently obtained by fireball networks, suggesting that Taurid bolides associated with the largest Encke meteoroids could produce meteorites and even might contribute significantly to the impact hazard (Madiedo et al. 2014; Napier 2010).

For the characteristics of the TC, we expect quite a heterogeneous progenitor, and as a consequence of its short orbital period as an evolved comet, we could also propose a brecciated nature due to collisional gardening (Trigo-Rodríguez 2015). Therefore, a diverse range of anomalous meteorites could be expected from a disrupted and brecciated object. Just as an example of the outcome of a body subjected to strong collisional processing, we could cite the heterogeneity found in the Almahatta Sitta ureilite body, which contains materials from many different rocky projectiles (Bischoff et al. 2010).

\subsection{Comparison with grouped carbonaceous chondrites}

Tubiana et al. (2015) found that the Encke reflectance spectrum is similar to the characteristics of primitive asteroids (Tubiana

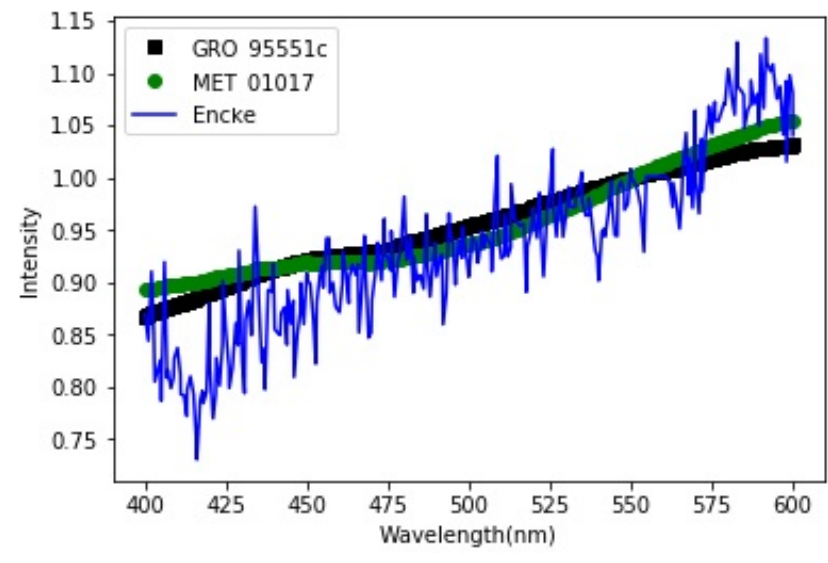

Fig. 2. Reflectance spectra from 400 to $600 \mathrm{~nm}$ of the two ungrouped carbonaceous chondrites compared with the spectrum of the comet 2P/Encke (from Tubiana et al. 2015).

et al. 2015), which are supposed to be correlated with CCs (Trigo-Rodríguez 2015). For that reason, we decided to establish a comparison with the rest of the $\mathrm{CC}$ spectra belonging to the chondrite groups of $\mathrm{CK}, \mathrm{CR}$, and CM. All the spectra that were obtained in the laboratory covered the 400-900 $\mathrm{nm}$ wavelength range and are included in Fig. 3.

CM chondrites probably originated from a hydrated C-rich asteroid (Trigo-Rodríguez et al. 2019; Rubin et al. 2007). Aqueous alteration of metal and silicate phases in that volatile-rich body have formed phyllosilicates and iron alteration minerals (Rivkin et al. 2002). Absorption band features around $700 \mathrm{~nm}$ in Fig. 3a are associated with hydrated minerals, so they could disappear after heating the material via, for example, collisional processing (Hiroi et al. 1996). As Tubiana et al. (2015) mentioned, looking at meteorite samples, lead us to believe that the surface spectra of comets and primitive asteroids may not be representative of their interiors. Despite the low overall reflectance, the other absorption features in the $380-450 \mathrm{~nm}$ region can indicate the presence of insoluble organic matter, or a metal-O charge transfer absorption (Cloutis et al. 2008), while the absorption features in the $450-480 \mathrm{~nm}$ range exhibit the presence of oxidised $\mathrm{Fe}$ species like hematite and goethite that produce an absorption band more extended than that of magnetite. The presence of an extended olivine band centred in $495 \mathrm{~nm}$ and visible in MET 01017 is also clear. The CM carbonaceous chondrites we studied here-Murchison and QUE 99355- are CM2 chondrites. According to the taxonomic classification scheme (Tholen 1984), there is a relation between the CM2 chondrites and asteroids, corresponding to an absorption band in the 700-750 $\mathrm{nm}$ region and an absorption feature near $900 \mathrm{~nm}$ (another absorption feature is in the $1100 \mathrm{~nm}$ region, although the spectral coverage of this wavelength region is not available). Aqueous alteration of metal and silicate phases in that volatile-rich body have formed phyllosilicates and iron alteration minerals (Zolensky \& McSween Jr 1988; Brearley \& Jones 1998; Krot et al. 2006; Alexander et al. 2012).

The CR chondrites belong to one of the major CC groups, with abundant free metal and magnetite that are a consequence of aqueous alteration (Kallemeyn et al. 1994; Cloutis et al. 2012a). Olivine is the dominant silicate in the CR2 and CR3 chondrites (Brearley \& Jones 1998). The spectral reflectance of the CR2 chondrite Renazzo shows that aqueous processes produce the alteration of olivine and pyroxene to phyllosilicates (Tholen 1984). Concerning the asteroid parent body of the CR hondrites, 

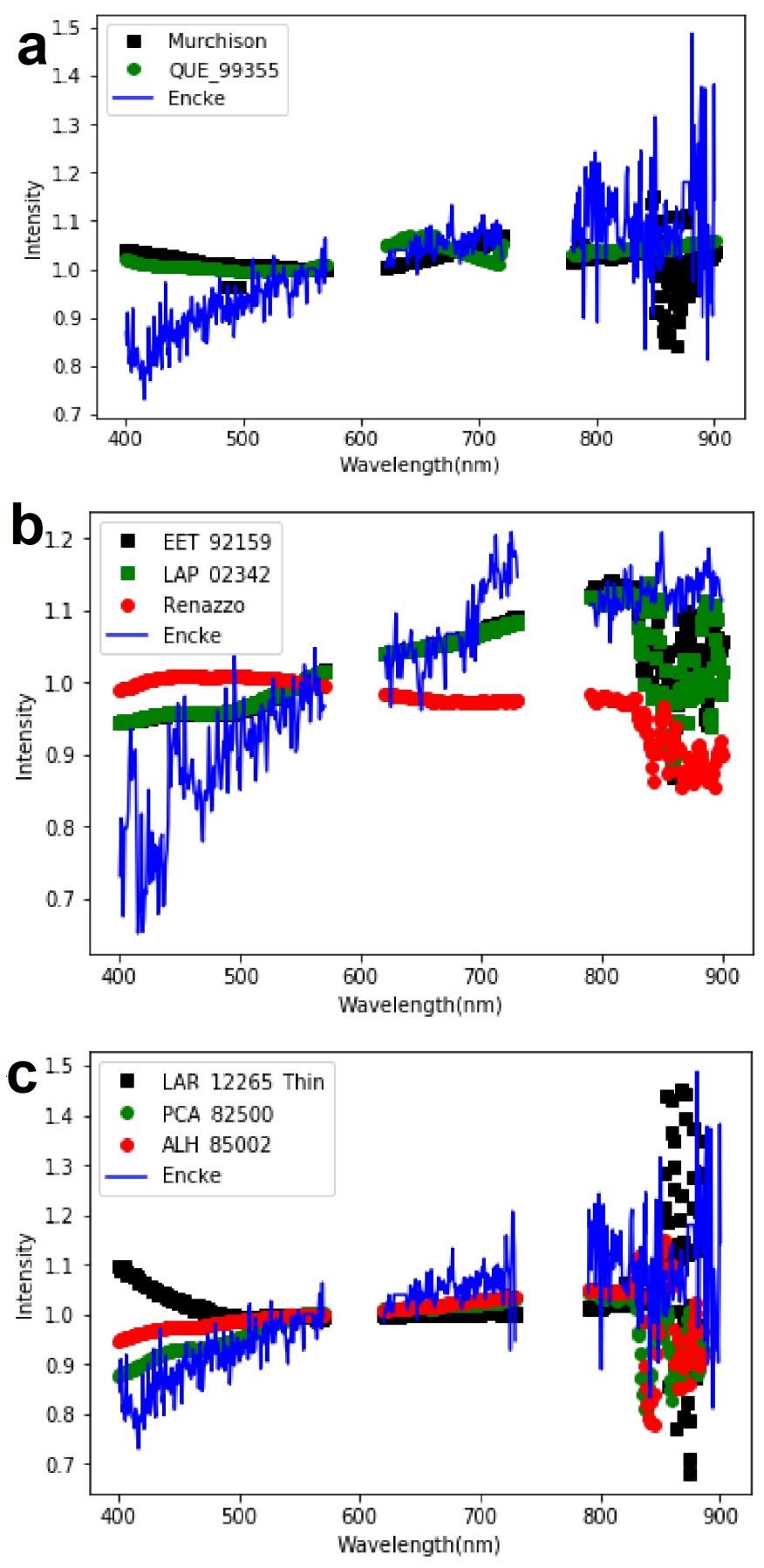

Fig. 3. Comparison of reflectance properties of comet $2 \mathrm{P} /$ Encke with selected specimens of the CC groups. Two CM2 chondrites are compared in $(a)$, three CR2 chondrites in $(b)$ and three CKs in $(c)$. All data were normalised to 1 at $550 \mathrm{~nm}$.

previous authors have not found any good match among the $\mathrm{C}, \mathrm{G}$, B, and F asteroids (Cloutis et al. 2012a; Hiroi et al. 1993, 1997). It is expected that the typical spectral features of the CR2 parent bodies are $\mathrm{Fe}^{2+}$ phyllosilicate, low-Fe olivine absorption features, a moderate overall reflectance $(\sim 8-12 \%)$, the presence of weak silicate absorption bands around 900 and $1100 \mathrm{~nm}$, a weak olivine absorption band at $900 \mathrm{~nm}$, and absent or quite weak $\mathrm{Fe}^{3+}-\mathrm{Fe}^{2+}$ phyllosilicate absorption bands in the $650-750 \mathrm{~nm}$ wavelength range (Cloutis et al. 2012a). The CR2 carbonaceous chondrites selected here to compare with the spectral properties of the comet Encke are EET 92159, LAP 02342, and Renazzo.
Figure $3 b$ clearly shows a very different spectral behaviour for the CR chondrites compared with Encke.

Concerning the spectral reflectance data for the CK group, we chose three specimens: LAR 12265 CK5, PCA 82500 CK4/5 and ALH 85002 CK5. This group is of interest here, because a previous comparison between CCs and the Eos asteroid family members provided a good match (Mothé-Diniz et al. 2008). Figure $3 \mathrm{c}$ exemplifies that there is probably not a compositional similitude between the comet Encke and the CK group. All the spectra of the CK meteorites show no shallow slope beyond $800 \mathrm{~nm}$ in contrast to Encke's spectrum, which is relatively flat beyond the $800 \mathrm{~nm}$ region.

Explaining how such extremely different objects as meteorites and comets may belong to the same TC is of utmost importance. It is well known that meteorites possess typical cohesive (tensile) strengths of several MPa (see e.g. Blum et al. 2006 for a compilation), whereas comets are extremely weak bodies. The latest estimate of the tensile strength of comets is only 1 Pa (Skorov \& Blum 2012; Blum et al. 2014, 2017; Attree et al. 2018) for length scales above $\sim 1 \mathrm{~cm}$, and $\sim 1 \mathrm{kPa}$ for length scales below that (Moyano-Cambero et al. 2016). The latter can be measured in meteor streams (Trigo-Rodríguez \& Llorca 2006, 2007), whereas the former is a requirement for the comet to become active (Gundlach et al. 2020). Besides this vast difference in cohesion, meteorites and comets also differ in porosity. While meteorites exhibit very low porosities of $\leqq 20 \%$ (Britt \& Consolmagno 2003), comets must be highly porous, with porosities of 60-80\% (Blum et al. 2006; Kofman et al. 2015; Pätzold et al. 2016; Fulle et al. 2016; Herique et al. 2019).

All primitive bodies in the Solar System date back to the formation era in which protoplanetary dust evolved into planetesimals. In recent years, evidence was gathered that comets in general (Skorov \& Blum 2012; Blum et al. 2014), the comet 67P/Churyumov-Gerasimenko (Blum et al. 2017; Fulle et al. 2016) in particular, and the Kuiper-Belt object (486958) Arrokoth (McKinnon et al. 2020) were formed through the gentle gravitational collapse of a cloud of $\mathrm{cm}$-sized "pebbles" (dust agglomerates). If we also apply such a formation mechanism for the progenitor of the members of the TC, we can state that its maximum size is constrained by several processes: (i) collisional destruction of the pebbles during the gravitational collapse, experimental and numerical work has shown that a maximum radius of the final object of $\sim 50 \mathrm{~km}$ is possible, before the pebbles would become collisionally fragmented (Jansson et al. 2017), which would in turn lead to a cohesive strength so high that dust activity would be impossible. (ii) Lithostatic destruction or deformation of the pebbles inside the planetesimal; given the cohesive strength of the pebbles of $\sim 1-10 \mathrm{kPa}$ (Blum et al. 2006) and the volume-average lithostatic stress inside a body of radius $\mathrm{R}$ of

$\frac{4}{15} \pi \rho^{2} G R^{2}$

with $\rho \approx 500 \mathrm{~kg} \mathrm{~m}^{-3}$ (Pätzold et al. 2016) and $G$ being the mass density of the body and the gravitational constant, we get stresses of

$4\left(\frac{R}{1 \mathrm{~km}}\right)^{2} \mathrm{~Pa}$.

Therefore, the maximum radius before the internal stresses destroy the pebbles is $R \cong 16-50 \mathrm{~km}$. However, even before the lithostatic stress can destroy the pebbles, the tensile strength may rise to values that would render activity impossible. It was 
shown experimentally that about $2.5-3 \%$ of the compressive stress remains in the body as tensile strength, even after the source for the stress has disappeared (Blum et al. 2014). Setting an upper limit of $1 \mathrm{~Pa}$ for the tensile strength of an active comet, we get an upper limit for the progenitor body of $R \cong 3 \mathrm{~km}$. We conclude here that the progenitor body of the Taurid complex and, thus, the comet Encke and the source of the meteorites, was a rather small body. As the fate of small bodies in the asteroid beltis to eventually be collisionally disrupted and reaggregated (Michel et al. 2001; Beitz et al. 2016; Schwartz et al. 2018), the progenitor planetesimal must have originated in a heavily underpopulated region of the young Solar System.

Although the modelling of the dynamical evolution from planetesimal to comet and meteorites is beyond the scope of this paper, we will try to sketch one possible scenario: during the evolutionary process of the Solar System, a high-velocity collision of the planetesimal with a much smaller impactor must have locally compacted its near-surface regions (Beitz et al. 2016, 2013). To achieve porosities $\leqq 20 \%$ (measured for meteorites Britt \& Consolmagno 2003), impact speeds of typically $1 \mathrm{~km} \mathrm{~s}^{-1}$ or above are required. If the impactor was sufficiently small, the collision was sub-catastrophic and the comet nucleus remained the largest intact post-collisional residue that suffered neither substantial compaction nor heating (Beitz et al. 2013), whereas sufficient compaction occurred at the impact site to create the low-porosity meteoritic matter (Beitz et al. 2016). Such an evolutionary scenario could be consistent with a significant number of ungrouped CCs available in meteorite collections (about 3\% of all CCs according to the Meteoritical Bulletin Database ${ }^{1}$ ). These ungrouped $\mathrm{CCs}$ are difficult to assign to $\mathrm{CC}$ groups because they have experienced significant thermal and aqueous alteration producing volatile element depletions and altering the organics forming their matrices (Alexander et al. 2007; Trigo-Rodríguez 2015), as we could expect to result from the heat and compaction produced by impact shock that in addition could explain their peculiar bulk elemental chemistry, with depletion of moderately volatile elements. In fact, the meteorite matrices exhibit the typical $1300 \mathrm{~cm}^{-1}$ disorder band assigned to graphite (see e.g. Larsen $\&$ Nielsen 2006). Whether such a scenario is plausible, or even likely, will be the task of future studies.

\section{Conclusions}

In our search for good meteorite proxies of short period comets, we compared the reflectance spectrum of the comet Encke with the spectral characteristics of several CCs. The absence of a clear spectral match between Encke and the previously cited CC groups might be not so surprising, given the diversity of rockforming materials that we expect form C-rich bodies in the Solar System. In that sense, the new results achieved by the OSIRISREx mission to asteroid (101955) Bennu clearly demonstrate that some asteroids are active sources of particles, probably evolving not very differently to the apparently inactive members of the TC (Lauretta et al. 2019). These so-called transitional asteroids produce meteoroid ejections and could possess evolved surfaces exhibiting further processing influenced by localized ice sublimation, phyllosilicate dehydration, thermal stress fracturing, and secondary impacts by their own ejected debris (Lauretta et al. 2019). Searching for ungrouped CCs that have experienced thermal processing makes sense as the reflectance spectra of evolved comets, like Encke, is modelled by the presence of thermally

1 https://www.lpi.usra.edu/meteor/metbull.php modified minerals like amorphous carbon, and mixtures of amorphous and crystalline silicates (Kelley et al. 2006). We find that the most common and well established aqueously altered CC groups do not match the spectrum of the comet Encke, but two anomalous meteorites might be good candidates to be associated with evolved comets. Consequently, we have reached the following conclusions:

1. Despite being almost featureless spectra, some spectral similarities between our two ungrouped $\mathrm{CC}$ and the spectrum of Encke were found. Olivine, phyllosilicates, and Fe oxides bands are present, and together with a similar spectral slope, they reasonably match the VIS-NIR reflectance spectrum characteristics of this short-period comet. In any case, we recognise that additional work is needed to establish a more precise link. Fresh meteorites or sample-returned materials unaffected by terrestrial weathering processes could be particularly useful in that regard.

2. Such a spectral match is not obvious for other CC groups. A comparison with $\mathrm{CM}, \mathrm{CR}$, and $\mathrm{CK}$ chondrites exhibits no evidence of compositional similarities with the comet Encke.

3. Further research into the reflectance spectra of other CCs could provide additional insight into the real nature, composition, and physical properties of periodic comets. Given the low albedo and overall dark nature of the surfaces of these objects, the characterisation of their rock-forming materials, which is currently being achieved by sample-return missions, is of seminal importance to mitigate future Earth encounters with these dangerous projectiles.

4. Finally, we think that a space mission to study the complex of bodies dynamically associated with the comet Encke or other short period comets is of key relevance. That mission should include sample return, to recognise and understand the materials forming not only this object, but also those probably active asteroids associated with the TC.

Acknowledgements. J.M.T.R. and S.T. acknowledge financial support from the Spanish Ministry (PGC2018-097374-B-I00, PI: JMTR). J.L.L. is grateful to ICREA Academia program and funding from Generalitat de Catalunya (2017 SGR 128). US Antarctic meteorite samples are recovered by the Antarctic Search for Meteorites (ANSMET) program which has been funded by NSF and NASA, and characterized and curated by the Department of Mineral Sciences of the Smithsonian Institution and Astromaterials Acquisition and Curation Office at NASA Johnson Space Center. We thank these institutions for providing the Antarctic meteorites studied here. J.B. thanks the Deutsches Zentrum für Luftund Raumfahrt (DLR) and the Deutsche Forschungsgemeinschaft (DFG) for their continuous support.

\section{References}

Alexander, C. O., Fogel, M., Yabuta, H., \& Cody, G. 2007, Geochim. Cosmochim. Acta, 71, 4380

Alexander, C. O., Bowden, R., Fogel, M., et al. 2012, Science, 337, 721

Asher, D., Clube, S., \& Steel, D. 1993, MNRAS, 264, 93

Attree, N., Groussin, O., Jorda, L., et al. 2018, A\&A, 611, A33

Babadzhanov, P., Williams, I., \& Kokhirova, G. 2008, MNRAS, 386, 1436

Beitz, E., Güttler, C., Nakamura, A., Tsuchiyama, A., \& Blum, J. 2013, Icarus, 225,558

Beitz, E., Blum, J., Parisi, M. G., \& Trigo-Rodriguez, J. 2016, ApJ, 824, 12

Belskaya, I., Bagnulo, S., Barucci, M., et al. 2010, Icarus, 210, 472

Binzel, R. P., Reddy, V., \& Dunn, T. 2015, Asteroids IV (Tucson, AZ: University of Arizona Press), 1, 243

Bischoff, A., Horstmann, M., Pack, A., Laubenstein, M., \& Haberer, S. 2010, Meteorit. Planet. Sci., 45, 1638

Blum, J., Schräpler, R., Davidsson, B. J., \& Trigo-Rodríguez, J. M. 2006, ApJ, 652,1768

Blum, J., Gundlach, B., Mühle, S., \& Trigo-Rodriguez, J. M. 2014, Icarus, 235, 156

Blum, J., Gundlach, B., Krause, M., et al. 2017, MNRAS, 469, S755

Boehnhardt, H., Tozzi, G., Bagnulo, S., et al. 2008, A\&A, 489, 1337 
Brearley, A. J. 1997, Science, 276, 1103

Brearley, A., \& Jones, R. 1998, Planetary Materials, ed., J. J. Papike (Washington, DC: Mineralogical Society of America), 3

Britt, D. T., \& Consolmagno, G. 2003, Meteorit. Planet. Sci., 38, 1161

Britt, D., \& Pieters, C. 1988, Lunar Planet. Sci. Conf. Proc., 18, 503

Burbine, T., Binzel, R., Bus, S., \& Clark, B. 2001, Meteor. Planet. Sci., 36, 245

Busemann, H., Alexander, M. O., \& Nittler, L. R. 2007, Meteorit. Planet. Sci., 42,1387

Cloutis, E. A., McCormack, K. A., Bell III, J. F., et al. 2008, Icarus, 197, 321

Cloutis, E., Hudon, P., Hiroi, T., Gaffey, M., \& Mann, P. 2011, Icarus, 216, 309

Cloutis, E., Hudon, P., Hiroi, T., \& Gaffey, M. 2012a, Icarus, 220, 586

Cloutis, E., Hudon, P., Hiroi, T., Gaffey, M., \& Mann, P. 2012b, Icarus, 221, 984

Cloutis, E., Hudon, P., Hiroi, T., et al. 2012c, Icarus, 221, 328

Cloutis, E. A., Pietrasz, V. B., Kiddell, C., et al. 2018, Icarus, 305, 203

Clube, S. V. M., \& Napier, W. M. 1984, MNRAS, 211, 953

Delbo, M., Libourel, G., Wilkerson, J., et al. 2014, Nature, 508, 233

Fulle, M., Della Corte, V., Rotundi, A., et al. 2016, MNRAS, 462, S132

Gradie, J., \& Veverka, J. 1986, Icarus, 66, 455

Gradie, J., Veverka, J., \& Buratti, B. 1980, Lunar Planet. Sci. Conf., 11, 357

Gundlach, B., \& Blum, J. 2016, A\&A, 589, A111

Gundlach, B., Fulle, M., \& Blum, J. 2020, MNRAS, 493, 3690

Herique, A., Kofman, W., Zine, S., et al. 2019, A\&A, 630, A6

Hiroi, T., Pieters, C. M., Zolensky, M. E., \& Lipschutz, M. E. 1993, Science, 261, 1016

Hiroi, T., Zolensky, M. E., Pieters, C. M., \& Lipschutz, M. E. 1996, Meteorit. Planet. Sci., 31, 321

Hiroi, T., Zolensky, M., \& Pieters, C. 1997, Lunar Planet. Sci. Conf., 28, 577

Hiroi, T., Zolensky, M. E., \& Pieters, C. M. 2001, Science, 293, 2234

Hsieh, H. H. 2017, Phil. Trans. R. Soc. A Math. Phys. Eng. Sci., 375, 20160259

Hsieh, H. H., \& Jewitt, D. 2006, Science, 312, 561

Jansson, K. W., Johansen, A., Syed, M. B., \& Blum, J. 2017, ApJ, 835, 109

Jewitt, D. 2008, in Trans-Neptunian Objects and Comets (Berlin: Springer), 1

Jewitt, D. 2012, AJ, 143, 66

Kallemeyn, G. 2000, Meteorit. Planet. Sci. Suppl., 35, A85

Kallemeyn, G. W., Boynton, W. V., Willis, J., \& Wasson, J. T. 1978, Geochim. Cosmochim. Acta, 42, 507

Kallemeyn, G. W., Rubin, A. E., \& Wasson, J. T. 1994, Geochim. Cosmochim. Acta, 58, 2873

Kelley, M. S., Woodward, C. E., Harker, D. E., et al. 2006, ApJ, 651, 1256

Kofman, W., Herique, A., Barbin, Y., et al. 2015, Science, 349, aab0639

Kresak, L. 1978, Bull. Astr. Inst. Czechosl., 29, 129

Krot, A. N., Hutcheon, I. D., Brearley, A. J., et al. 2006, Meteorites and the Early Solar System II, 525 (Tucson, AZ: University of Arizpna Press)

Larsen, K., \& Nielsen, O. F. 2006, J. Raman Spectr., 37, 217

Lauretta, D., Hergenrother, C., Chesley, S., et al. 2019, Science, 366, 1217

Levison, H. F., Terrell, D., Wiegert, P. A., Dones, L., \& Duncan, M. J. 2006 Icarus, 182, 161
Luu, J., \& Jewitt, D. 1990, Icarus, 86, 69

Madiedo, J. M., Ortiz, J. L., Trigo-Rodríguez, J. M., et al. 2014, Icarus, 231, 356 McKinnon, W., Richardson, D., Marohnic, J., et al. 2020, Science, 367, eaay6620 Metzler, K. 2012, Meteorit. Planet. Sci., 47, 2193

Michel, P., Benz, W., Tanga, P., \& Richardson, D. C. 2001, Science, 294, 1696

Micheli, M., Wainscoat, R. J., \& Denneau, L. 2018, Icarus, 303, 265

Mothé-Diniz, T., Carvano, J., Bus, S., Duffard, R., \& Burbine, T. 2008, Icarus, 195, 277

Moyano-Cambero, C., \& Trigo-Rodríguez, J. 2015, Lunar Planet. Sci. Conf., 46, 1106

Moyano-Cambero, C. E., Trigo-Rodríguez, J. M., Llorca, J., et al. 2016, Meteorit. Planet. Sci., 51, 1795

Muinonen, K., Belskaya, I. N., Cellino, A., et al. 2010, Icarus, 209, 542

Müller, T., Durech, J., Ishiguro, M., et al. 2017, A\&A, 599, A103

Napier, W. M. 2010, MNRAS, 405, 1901

Pätzold, M., Andert, T., Hahn, M., et al. 2016, Nature, 530, 63

Popescu, M., Birlan, M., Nedelcu, D., Vaubaillon, J., \& Cristescu, C. 2014, A\&A, 572, A 106

Raponi, A., Ciarniello, M., Capaccioni, F., et al. 2020, Nat. Astron., 4, 500

Rivkin, A., Howell, E., Vilas, F., \& Lebofsky, L. 2002, Asteroids III (Tucson, AZ: University of Arizona Press), 1, 235

Rubin, A. E., Trigo-Rodríguez, J. M., Huber, H., \& Wasson, J. T. 2007, Geochim. Cosmochim. Acta, 71, 2361

Schwartz, S. R., Michel, P., Jutzi, M., et al. 2018, Nat. Astron., 2, 379

Scott, E., \& Krot, A. 2003, Treatise Geochem., 1, 711

Sekanina, Z. 1998, Planet. Space Sci., 46, 191

Skorov, Y., \& Blum, J. 2012, Icarus, 221, 1

Spurnỳ, P., Borovička, J., Mucke, H., \& Svoreň, J. 2017, A\&A, 605, A68

Tanbakouei, S., Trigo-Rodríguez, J. M., Sort, J., et al. 2019, A\&A, 629, A119

Tholen, D. 1984, Asteroid taxonomy from cluster analysis of Photometry, Ph.D. thesis, University of Arizona, Tucson

Trigo-Rodríguez, J. M. 2015, Planet. Mater. EMU Notes Mineral., 15, 67

Trigo-Rodríguez, J. M., \& Llorca, J. 2006, MNRAS, 372, 655

Trigo-Rodríguez, J. M., \& Llorca, J. 2007, MNRAS, 375, 415

Trigo-Rodríguez, J. M., \& Williams, I. P. 2017, in Assessment and Mitigation of Asteroid Impact Hazards (Berlin: Springer), 11

Trigo-Rodriguez, J. M., Moyano-Cambero, C. E., Llorca, J., et al. 2014, MNRAS 437, 227

Trigo-Rodríguez, J. M., Rimola, A., Tanbakouei, S., Soto, V. C., \& Lee, M. 2019, Space Sci. Rev., 215, 18

Tubiana, C., Snodgrass, C., Michelsen, R., et al. 2015, A\&A, 584, A97

Weisberg, M. K., Prinz, M., Clayton, R. N., et al. 2001, Meteorit. Planet. Sci., 36, 401

Weisberg, M. K., Ebel, D. S., Nakashima, D., Kita, N. T., \& Humayun, M. 2015, Geochim. Cosmochim. Acta, 167, 269

Zolensky, M., \& McSween Jr, H. Y. 1988, Meteorites and the early solar system, 114 